\title{
LA CIENCIA Y TECNOLOGÍA CON PERSPECTIVA DE GÉNERO.
}

\section{SCIENCE AND TECHNOLOGY FROM A GENDER PERSPECTIVE}

Patricia García Guevara[1]

\begin{abstract}
RESUMEN
La participación femenina en las ciencias exactas y las ingenierías adopta un ordenamiento peculiar en lo educativos y segregacionista en las asociaciones y en laboral. En este trabajo nos proponemos abordar esta problemática. Empleamos un enfoque que incorpora la sociología de la ciencia y una perspectiva de género con el que exploramos cuáles son los "nichos científicos" en que les ha sido posible incursionar para el logro y desempeño profesional. Los resultados provienen de un estudio cualitativo. Aquí utilizamos datos provenientes de entrevistas a académicas y profesionales que nos dan cuenta de cómo su ejercicio profesional es una red que entrelaza sus historias personales con las instituciones sociales involucradas en el desarrollo científico.
\end{abstract}

Palabras claves: Género, ciencia y tecnología, profesión.

\begin{abstract}
Women's participation in the hard sciences and engineering adopts a peculiar ordering in the educational and segregationist in associations and labor. In this work we will tackle this problem. We use an approach that incorporates the sociology of science and a gender perspective which explores what are the «scientific niches» that have been possible to break and their achievement and professional performance. The results come from a qualitative study. Here we use data from interviews with academics and practitioners that give us how their practice is a network that weaves their personal stories with social institutions involved in scientific development.
\end{abstract}

Keywords: Gender, science and technology, profession

\section{INTRODUCCIÓN}

La participación piramidal de las mujeres en las ciencias exactas y las ingenierías nos expresa estadísticamente la punta de un iceberg. De entrada nos sitúa cuantitativamente respecto a un problema más profundo. Por lo mismo nos vemos obligadas a indagar en otros espacios además del sistema educativo, en el mercado laboral, en las asociaciones profesionales y hasta en algunas de las instancias burocráticas estatales relacionadas con la ciencia y la tecnología. Por ejemplo, al asomarnos a la industria de la electrónica en la ciudad mexicana de Guadalajara, observamos a empresas de manufactura que cuentan con porcentajes hasta de un $80 \%$ de obreras. En contraste con la industria farmacéutica, en donde las cifras en puestos altos de profesionistas son nulas o casi nulas, aún cuando el egreso universitario en carreras como la de Qímica-Farmacobióloga y la Licenciatura en Química se han feminizado con egresos mayores al 50\% (García Guevara, 2006a).

№: 23-24 Ano: 13 jul/ago/set/out/nov/dez 2011 
Egreso femenino en algunas carreras de ingeniería y ciencias exactas

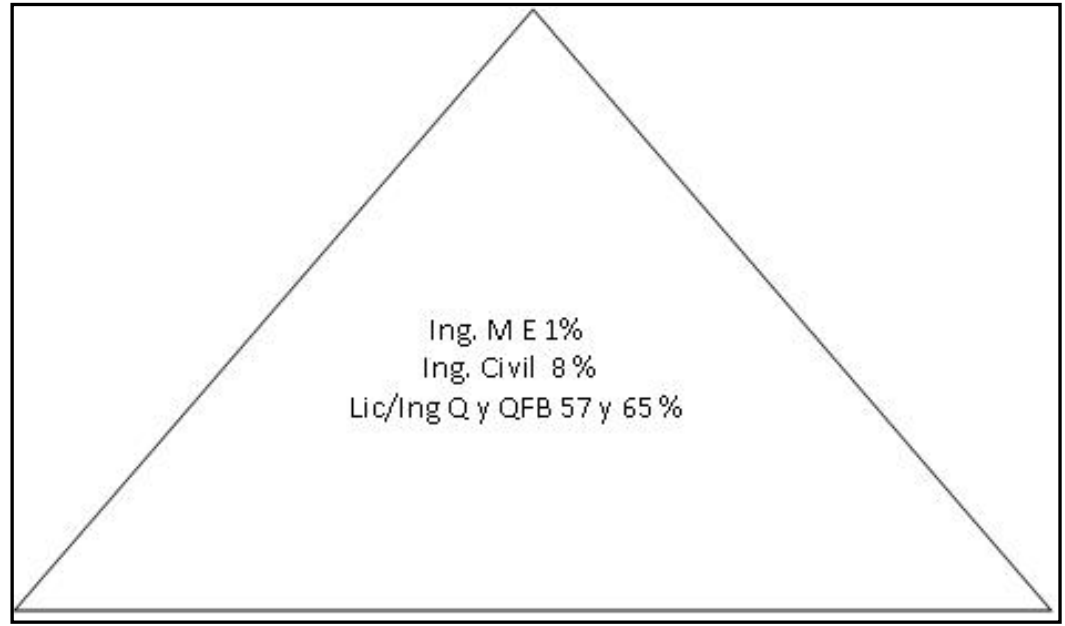

Participación de las científicas en la Academia Mexicana de las Ciencias

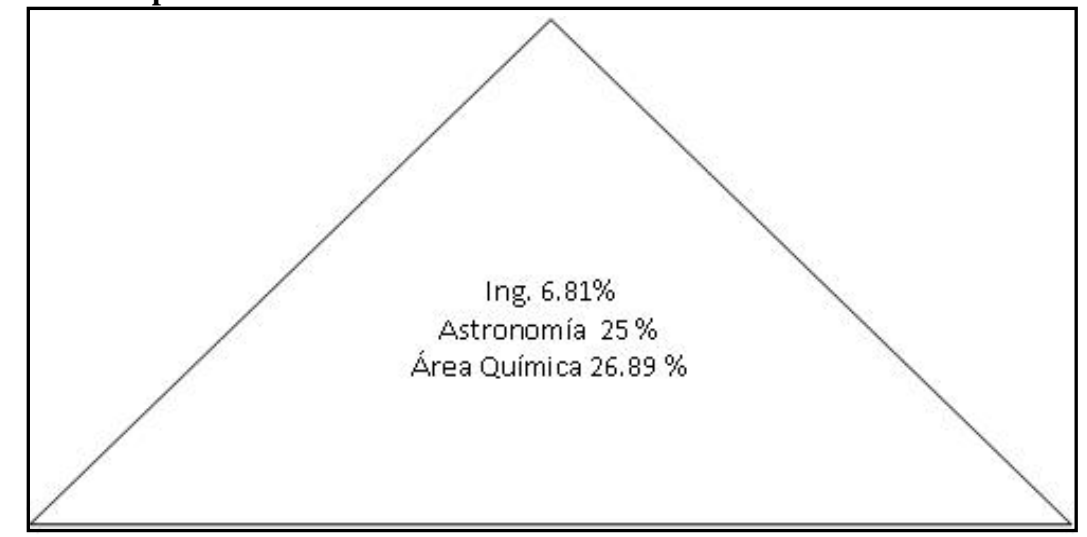

Participación femenina en la industria farmacéutica y de la electrónica

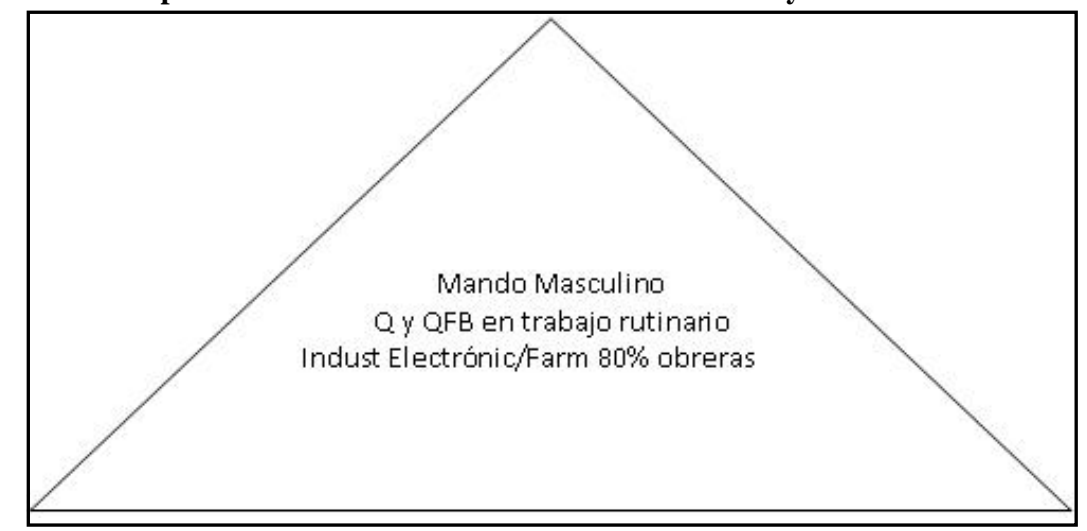

№: 23-24 Ano: 13 jul/ago/set/out/nov/dez 2011 
Estamos frente a un problema complejo que involucra varias partes. Abordar esto, requiere de una visión amplia, un enfoque multidisciplinario entre la sociología de la ciencia y una perspectiva de género que nos dé cuenta de la complejidad del mismo. Explicar, cuestiones como cuáles son los "nichos científicos" en que les ha sido posible incursionar a las mujeres científicas dentro de una cultura patriarcal que inhibe de manera velada el desempeño de las mismas, es algo complicado. Aquí nos proponemos estudiar el proceso mismo de la profesión científica a partir del prestigio y la experiencia profesional que algunas mujeres han logrado. Los resultados provienen de un estudio cualitativo parcial que forma parte de una investigación más amplia. Aquí utilizamos datos provenientes de entrevistas a profesoras-investigadoras de cuatro instituciones universitarias: UNAM, IPN, la Universidad de Guadalajara, la Universidad Autónomas de Monterrey, de la industria farmacéutica y una funcionaria del IMPI. Las entrevistadas nos dan cuenta de cómo el ejercicio profesional es una red de prácticas que se entrelazan con las historias personales y sociales de las instituciones involucradas en el desarrollo científico.

La distribución por sexo en las ciencias exactas y las ingenierías, nos sirve como una primera base para buscar el por qué de esta conformación con estudios de tipo cualitativo[2]. Estamos frente a una ordenación cambiante, es decir, es pausada en la mayoría de esas carreras ingenieriles y en otras pocas es sostenida. Por lo mismo, la estrategia sociológica para abordarlas implica tomar en cuenta distintas vertientes. Las interconexiones que prevemos son complejas y nos proponemos prestar particular atención a las siguientes cuestiones (Fox-Keller, 1989; García Guevara, 2007):

a) el desarrollo tecnológico y educativo del país en general, y en particular de las mismas disciplinas, la innovación y su relación particular con estrategias que incluyen o excluyen abierta o veladamente la participación femenina (cfr. García Guevara, 2006),

b) el grado de expertise y el capital cultural como estudiantes y/o científicas,

c) la clase social,

d) el proceso de socialización en el que como estudiantes estuvieron inmersas. Nos referimos al contexto cultural patriarcal mexicano pero también a las historias particulares de las mismas mujeres.

e) las políticas públicas estatales hacia la ciencia y la tecnología en general,

f) y en particular, la gestión de políticas específicas como la equidad de género en las instituciones educativas y laborales.

Esta estrategia de abordaje, trata de ir más allá de las descripciones lineales y cuantitativas de género hacia el análisis de procesos amplios, dado que, las características particulares personales de las científicas se entrecruzan con la cultura y el desarrollo estructural del contexto mexicano.

\section{EL DESARROLLO CIENTÍFICO, LA BUROCRACIA Y LAS POLÍTICAS PÚBLICAS.}

México no es un país altamente industrializado como los países del hemisferio norte, sin embargo, sí posee algunas regiones o enclaves con tecnología de punta, como lo son la frontera norte, la zona centro y centro occidente del país[2]. Toda empresa industrial de cualquier ramo, depende del desarrollo de la ciencia, la tecnología y de la también llamada innovación. El problema de ésta última, es que descansa en una relación triangular que conforman el sistema de educación, el sector empresarial y el Estado[3]. El buen

№: 23-24 Ano: 13 jul/ago/set/out/nov/dez 2011 
funcionamiento de este triángulo es indispensable para que se dé la producción, el crecimiento y la retroalimentación del desarrollo tecnológico y científico. Las siguientes citas nos ejemplifican sobre las distintas vertientes del problema desde la experiencia profesional de algunas de las científicas mexicanas.

\section{EUREKA: INNOVACIÓN Y RETROALIMENTACIÓN}

"Originalmente yo solamente estaba pensando en encontrar moléculas de las amibas involucradas en daño con la estrategia que diseñé para ese fin... Mi trabajo lo presenté en un congreso sobre amibiasis y fui duramente criticada... Estas duras críticas, me obligaron a buscar explicar porqué con la metodología que estaba usando, las moléculas no se degradaban... en menos de 15 días comprobé con la metodología que estaba usando la actividad de las enzimas era menor que la observada con los inhibidores. Le platiqué esto al Dr. X y me propuso buscar una patente ... el me proporcionó todo el apoyo, me presentó al dueño de uno de los despachos de abogados más prestigiosos para que me ayudaran... tengo dos patentes en México y en Estados Unidos. Mi trabajo actual quizás obtendrá dos patentes más..."

En el ejercicio profesional de las investigadoras, los hallazgos originales o descubrimientos científicos y la reproducción de éstos a gran escala las han llevado a enfrentar lo que se le denomina el registro de su invento y/o marca ante el Estado. Las distintas entrevistas muestran que estamos frente a un proceso dificultoso, dadas las características administrativas del Estado mexicano y de los corporativos trasnacionales. El Instituto Mexicano de Propiedad Intelectual (IMPI)[4] es instancia creada en 1994[5] que cuenta con una ley, normas, reglas e instituciones federales y descentralizadas al interior del país. La práctica de la reglamentación se ven reflejadas en una tramitología burocrática y un funcionamiento organizativo particular que mostramos abajo en palabras de las mismas investigadoras.

\section{BUROCRACIA: TRÁMITES, FORMAS Y VENTANILLAS}

“[El proceso] es muy complicado, el trámite tarda como dos años. Además el costo es muy superior en la patente que en la marca, requieres de mucha asesoría del personal del IMPI porque es muy especializado, ya que tienes que describir el invento, hacer resumenes, reivindicación, entre otros. La redacción y dibujos son muy específicos. Se deben respetar desde márgenes, tipos de dibujos, el resumen por citar algunos... Existe un trámite para que veas si tu patente no está registrada en otra parte del mundo o en México... Si quieres proteger en otro país tienes que hacerlo país por país... La complejidad y el tiempo tarda... En México se otorgan al año cerca de 5000 patentes y el $90 \%$ son extranjeras. Mientras que en Japón o en Estados Unidos las 5,000 se registran en un día. Además en E.U. tu puedes patentar lo que tu quieras" (Entrevista funcionaria IMPI, 2007).

Vale la pena contrastar esta normatividad oficial en la vida diaria. Es decir, en la práctica cómo enfrenta esto una pequeña empresaria QFB con más de 30 años en el ramo de los farmacéuticos en una de estas instancias gubernamentales. La Secretaría de Salud Pública también está relacionada con la producción científica para el caso del registro de marcas y

№: 23-24 Ano: 13 jul/ago/set/out/nov/dez 2011 
del funcionamiento de los laboratorios privados. Abajo citamos en extenso este proceso ya que nos transcribe la magnitud de la problemática que va aparejada al desarrollo y producción científica y tecnológica en estas mujeres. Los detalles nos ilustra sobre una amplia gama de cuestiones que van desde la ineficiencia burocrática, la corrupción nacional hasta la guerra de patentes entre los corporativos y el espionaje industrial, etc.

"yo nunca quise entrar a hacer una línea propia de productos... un cliente ya tenía su propia marca de productos y comencé a maquilarle... en la actualidad tenemos 6 productos, son cápsulas y una crema... También dedico mi tiempo a hacer trámites en Salubridad... las personas que están en ciertos puestos aquí en Guadalajara en el sector salud, son todo menos químicos, un médico, un veterinario, una enfermera... Normalmente cada 2 o 3 años vienen de México y dan una conferencia de legislación farmacéutica y te dicen lo último que están pidiendo... los jefes de aquí mandan a unos cuantos no a todos... así el jefe no tiene ni la menor idea y entre ellos hay diferencias. En el D. F. también hay criterios personales. Tu vas y entregas tu papelería y dependiendo de la persona que te recibe los papeles, te dicen "no aquí cámbiamelo, me le vas a poner esto". Te sales, te vas, si regresas y te toca otra persona en otra ventanilla, te dice "no, está mal, tienes que ponerle esto", y es como tu lo habías llevado originalmente... los trámites estatales son más pachanga, acá te tardas meses y a cada ratito vas a tener una inspección... por ejemplo, a mi me llegaba una visita de Salubridad Federal y me decía: "aquí quiero una puerta la próxima vez que venga y regreso en uno, tres meses o un año". Tú sabías que tenías que tener la puerta para esa fecha. Pero resulta que en cuanto la tenías llegaba la visita estatal y te decían "y esa puerta que hace aquí ... no quiero esa puerta y me la tapas". Aunque digan que no hay corrupción, es nada más para sacar mordida, todo mundo tiene su precio .... Si yo digo que voy a registrar una patente para hacer guacamole, entonces te encuentras que hay gente muy lista que se dedica a ver las patentes que ya están registradas. Entonces llegan y registran tu patente, pero en lugar de ponerle cebolla blanca le ponen cebolla morada y ya fue otra patente completamente diferente, aunque a ti te costo trabajo sacar la fórmula del guacamole, con cualquier elemento que le varíen ya es otra patente. Realmente no te sirve de mucho registrar tu patente aquí en México no te respetan... En cuestión de la industria farmacéutica se supone que si tienes una patente de las sales de medicamentos nuevos por 20 años no la pueden explotar otros. Pero ahorita hay un problema fuerte, creo que es con Bayer, la matriz Alemania, está sacando el producto en Brasil con otro nombre de la sal y es la misma sal, con una pequeña variación..."

\section{LA GESTIÓN DE LAS POLÍTICAS PÚBLICAS CON PERSPECTIVA DE GÉNERO}

La anterior entrevista muestra la forma triangular en que se sostiene el desarrollo científico y tecnológico: educación, empresa y las distintas instituciones del Estado involucradas. Por lo mismo algunos autores han llegado a denominarla como una relación tripartita que en nuestro país se ha convertido en una relación "viciosa". En contraste con otros países que han logrado hacer funcionar ese triangulo de manera "virtuosa" (Casas,

№: 23-24 Ano: 13 jul/ago/set/out/nov/dez 2011 
2003; García Guevara, 2006b). En todo caso, el texto de arriba, nos evidencia la naturaleza social de la ciencia y su complejidad, tanto como los distintos actores involucrados en el proceso de la ciencia y la tecnología y su progreso. Las tres instituciones involucradas socialmente están obligadas a enfrentar la sub-representación de las mujeres en las ciencias exactas e ingenierías con políticas públicas que lleven a buen término la aplicación de la Ley de Equidad.

Si bien, ésta apenas se comienza a hacer presente en algunas empresas nacionales y trasnacionales como la HP, IBM y Solectrón, mediante la firma de convenios específicos[7] que ha impulsado el Instituto Nacional de las Mujeres; esto no es todavía una realidad en la pequeña industria o en algunas trasnacionales estadounidense de capital Republicano que son más renuentes a las políticas afirmativas de equidad de género para promover ingenieras en puestos altos (García Guevara, 2006a). Menos aún, podríamos hablar de la implementación de un sistema de monitoreo que le den seguimiento a los acuerdos pactados por las empresas. Por ejemplo, a nivel nacional han participado con la puesta en marcha de esta política con perspectiva de género, empresas como, Kraft Food, Wyn de México Productos Químicos S.A. de C.V., Eli Lilly de México, Bristol-Myers de México, Corporación Manufacturera de Electro Equipos S.A. de C.V., etc. (cfr. Instituto Jalisciense de las Mujeres).

\section{EL RECONOCIMIENTO CIENTÍFICO}

El fenómeno de las prácticas masculinas en términos de estrategias de exclusión o de inclusión en el ordenamiento y canalización hacia profesiones de menor prestigio y remuneración por género, ya comienza a ser documentada por diversos estudios en el país[8]. Sin embargo, tenemos muchos vacíos en las investigaciones para perfilar una discusión más amplia sobre los cambios recientes de las científicas mexicanas[9]. Sabemos muy poco de los descubrimientos científicos originales que han hecho las mexicanas con alto perfil y el proceso interactivo con los grandes corporativos industriales que acompaña a la consecución del prestigio científico[10].

En algunas de mis entrevistas, sobresalen dos elementos para el caso particular del área de la física y alguna ingeniería. En primer lugar, resalta la clase social. En segundo lugar, encontramos un fuerte trabajo en redes científicas vinculadas a la industria. En ambos casos el proceso de socialización desde su temprana edad ha sido clave. En ellas encontramos familiares como padres científicos, maestros o parientes que veían positivamente las destrezas femeninas en las matemáticas o con la imaginación creativa de las futuras científicas.

En mis entrevistas, algunas mujeres ingenieras que han logrado cierto renombre y alcanzado puestos altos, encontré en dos de ellas, a padres con un reconocido prestigio científico. Aquí, es fácil ver la manera cómo esto fue ventajoso para ellas, el ser herederas de un capital cultural y de apoyo socio-económico. Tampoco parecen cuestionar los cánones masculinos adversos a su desempeño, tal vez porque su carrera e inserción en puestos altos estuvo mediatizada precisamente por las relaciones de clase y una ideología del "esfuerzo individual", o de "abeja reina" que "amortigua" la competencia masculina.

Hay otro pequeño grupo de ingenieras que el reconocimiento de sus logros ha sido ganado mediante una lucha contra corriente y que podríamos denominar "batalla profesional".

№: 23-24 Ano: 13 jul/ago/set/out/nov/dez 2011 
En sus trayectorias profesionales destaca la falta de un capital social, cultural o económico que no heredaron. Nos referimos a la pertenencia a asociaciones científicas, al trabajo de cabildeo y el establecimiento de redes de financiamiento con ejecutivos o dueños de la industria, rectores o con "vacas sagradas" científicas de universidades nacionales o extranjeras. Todos estos componentes mezclados las han llevado a la consecución de grandes proyectos científicos. Aunque se dice fácil, ha sido una labor ardua de muchos años, sobre todo si consideramos que se trata además de redes con predominio masculino que se entrelazan y convencer a tales audiencias requiere erosionar el monolito cultural patriarcal en muchos sentidos.

¿Que involucra el desarrollo de estas tareas, que por su parte son un componente fundamental en las trayectorias exitosas de científicos hombres y mujeres? Encontramos que requiere de varios elementos. Por ejemplo, contar con un alto grado de "expertise" -conocimiento en su área de estudio- por medio de doctorados. Exige el desarrollo de habilidades políticas y de un complicado trabajo de cabildeo. También un trabajo destinado a la formación de redes científicas y vinculación para conseguir presupuesto y esto último es algo muy complejo; ya que implica el desarrollo específico de proyectos de investigación que generen resultados concretos y que interesen tanto a las universidades como a la industria.

En suma, podremos resaltar algunos de estos elementos en este aporte de discusión: se trata de un largo y extenuante trabajo que requiere de dos cuestiones: Primero, convencer a ejecutivos de la industria, rectores universitarios y un equipo de ingenieros que provean de financiamiento, espacios en laboratorios ya sea en la misma industria y/o en universidades. Generar tales espacios es costoso[11] y en muchas ocasiones hay que trabajar convenios para la procuración de los mismos.

"Siempre he trabajado con el apoyo brindado por la UANL. Aunque siempre he tenido que trabajar mucho y con poco dinero. Durante muchos años mis proyectos fueron rechazados por CONACYT[12]... he tenido que convencer a la gente, he tenido que ir abrir puertas, explicar, hacer antesalas. He recibido golpes bajos. Dolorosamente he perdido personas que yo consideraba amigos. He tenido que aprender también a base de golpes duros u decepciones, que a la mayoría de las personas con dinero en el mundo, no les interesa las enfermedades de la gente pobre".

En segundo lugar, el mercado globalizado de hoy en día, requiere de un alto grado de conocimiento y que los científicos involucrados estén dispuestos al intercambio de resultados. Esto último, cuestiona muchos esquemas tradicionales de trabajo y autoría. Romperlos y que los científicos estén dispuestos a socializar sus hallazgos científicos con sus pares es algo a lo que tienen que estar dispuestos los científicos para que el conocimiento fluya. La industria local hace firmar a sus estudiantes que trabajan en sus proyectos incubadoras sobre los hallazgos que surjan de dichos experimentos como "secreto industrial". Sin embargo, la burocracia de patentes, marcas, el espionaje y la corrupción es algo que también requiere se documentada.

№: 23-24 Ano: 13 jul/ago/set/out/nov/dez 2011 


\section{CONCLUSIÓN}

A manera de conclusión podemos decir que las condiciones actuales con vertiginosos avances en la ciencia y la tecnología dan lugar a la emergencia de nuevas formas de conocimiento tecnológico a partir de la innovación. Esto obliga, por un lado, a otro tipo de organización del mismo, como los proyectos incubadora y exige otros comportamientos derivados de éste. En el caso de las universidades la inclusión de nuevos comportamientos más allá de la transmisión "pastoral" fuera de las aulas y la inclusión de talentos sin importar el sexo de las personas sino su "expertise" o capacidad. Por el otro, nuevos esquemas burocráticos por parte del Estado, los funcionarios y servidores público. Lo mismo aplica al investigar a la cultura científica globalizada y sus efectos específicos por género.

La perspectiva sociológica que aborda los procesos a partir de las partes involucradas, las referencias institucionales a las que se enfrentan, las negociaciones y recursos que entran en el intercambio, la movilización de recursos heterogéneos, las reglas y las normas, las continuas interacciones que se dan entre las partes involucradas: la ciencia, la tecnología, el mercado, las universidades, las políticas de Estado y la comunidad científica (Santos y de Gortari, 2003). Es decir un abordaje a partir de la complejidad.

El problema de esta propuesta es que el enfoque sociológico de las profesiones como las ingenierías no toman al género como eje de explicación. Las cifras nos pueden distraer por los pequeños incrementos de mujeres en las ciencias exactas y las ingenierías. Sin embargo, necesitamos discutir las transformaciones que ha generado por ejemplo, la industria y que espacios se resisten al cambio para incluir el talento femenino, la documentación de experiencias de gestión efectivas con perspectiva de género, en relación a los premios científicos, nuevas patentes y proyectos incubadoras que involucran a mujeres.

\section{NOTAS}

[1] Universidad de Guadalajara.

[2] Fox-Keller fue una de las pioneras en este tipo de estudios en México hay poco estudios en este campo.

[3] Solamente la industria de la electrónica de alta tecnología representó en 2004 casi el 25\% del total de las exportaciones en México. Se trata del llamado Valle del Silicón Mexicano localizado en el estado de Jalisco (García Guevara 2006b).

[4] Léase apoyo, visión, democratización, etc.

[5] Lamentablemente esta funcionaria no me pudo proporcionar estadísticas de patentes por sexo. La institución no ha generado ni un sistema, ni considera la utilidad social de un desglose de las cifras. Contabiliza las solicitudes de patentes o registro de marcas por usuarios: globales por empresas, inventores, investigadores, unidades académicas o personas físicas.

[6] En 1999 el estado mexicano, decreta la Ley de Fomento a la Investigación Científica y Tecnológica.

[7] Acuerdos impulsados por Instituto Jalisciense de la Mujer en el estado el 7 de agosto de 2006 apoyados por las cámaras nacionales de la industria como CANIETI y CADELEC.

[8] Para el caso de la temprana feminización de profesiones como la de químico-farmacobiólogía, su explicación está relacionada con dos cuestiones: a) su ethos light y b) la inclusión o "anuencia" patriarcal: espacios cerrados, bajo la custodia paterna, utilizando matraces y material liviano, con carácter asistencial, etc. En contraposición, tenemos a la ingeniería civil

№: 23-24 Ano: 13 jul/ago/set/out/nov/dez 2011 
con un ethos rural pesado: con uso de maquinaria pesada, entre el polvo y la tierra (cfr. García Guevara, 2006a).

[9] Recientemente algunas académicas se han dado a la tarea de rescatar biografías de científicas mexicanas (cfr. Carega, 2008).

[10] Aunque Conacyt ha tratado de visibilizar algunos de los grandes descubrimientos de las científicas mexicanas en sus boletines, o incluso es sus "spots" telefónicos; esta "visibilización” o "discriminación positiva" es muy escueta.

[11] Sean el caso de laboratorios del sector público como las universidades o como empresa privada.

[12] Consejo Mexicano de Ciencia y Tecnología.

\section{BIBLIOGRAFÍA}

Martha Caballero y Patricia García Guevara (2007). Género, cultura y sociedad. Serie de investigaciones del PIEM: Curso de vida y trayectorias de mujeres profesionales. México: El Colegio de México.

Casas, Rosalba (2003). "Los estudios sociales de la ciencia y la tecnología: enfoques, problemas y temas para una agenda de investigación”, en María Josefa Santos Corral (coord.) Perspectivas y desafíos de la educación, la ciencia y la tecnología. México: UNAM/IIS.

Fox Keller, Evelyn (1989). Reflexiones sobre género y ciencia. España: Ed. Alfons el Magnanim.

García Guevara, Patricia (2006a). "Masculinización y feminización de las profesiones tradicionalmente masculinas: El caso de las ingenierías", en Roberto Miranda Guerrero et al (coord.) Hombres y masculinidades en Guadalajara. México: Universidad de Guadalajara.

García Guevara, Patricia (2006b). "Nuevas y viejas dinámicas para la ciencia, la tecnología y la educación superior", en María Alicia Peredo Merlo et. al (coord.) Tendencias de la educación superior en México. México: Universidad de Guadalajara.

Santos, María Josefa y Rebeca de Gortari (2003). "De contactos a redes: La construcción de redes de conocimiento a través de la formación de recursos” en Matilde Luna (coord..) Itinerarios del Conocimiento: Formas, Dinámicas y Contenido. México: Antropos-UNAM. 
Cadernos de Gênero e Tecnologia 